\title{
Research on Direct Epoxidation of Propylene over Modified Au/Ts-1
}

\section{Catalysts}

\author{
Lina Wang ${ }^{1, a}$ \\ ${ }^{1}$ Xi an University of Science and Technology, Xi'an, Shaanxi, China, 710054 \\ ${ }^{\mathrm{a}}$ email
}

Keywords: Modified Au/Ts-1, Direct Epoxidation, Propylene Epoxidation

\begin{abstract}
Currently, the $\mathrm{C}_{3} \mathrm{H}_{6}$ conversion is usually low on the $\mathrm{Au} / \mathrm{TS}-1$ system. Therefore, the improvement of $\mathrm{C}_{3} \mathrm{H}_{6}$ conversion is vital to the commercialization. A series of Au catalysts supported on modified Au/TS- 1 were prepared and tested for direct propylene epoxidation in the presence of hydrogen and oxygen. The effect of In contents on the structure and catalytic properties of these catalysts were investigated in the paper to provide some references for the relative researchers.
\end{abstract}

\section{Introduction}

Sn (IV) was doped in the synthesis of TS-1 (IV), and Sn ions were found to enter the molecular sieve framework. The Au catalyst prepared by this carrier is used for the direct oxidation of propylene by gas phase epoxidation, which effectively improves the conversion ratio and PO selectivity of propylene. We know that the TS-1 molecular sieve is a typical structure of MFI microporous molecular sieve. Most of the Ti (IV) is strictly in four-coordination-state, and the theory research shows that the saturated Ti species name, on the propylene epoxidation activity is very low, unsaturated Ti species coordination catalytic activity significantly increased. Therefore, it is very advantageous to increase the oxidation activity of the Ti species on the carrier. The catalytic activity of TS-1 catalyst for epoxidation of propylene can be improved by modifying the carrier TS-1.

This research comes from the Yang's inspiration. They used In modified Au/APTMS-SBA-15 catalyst for liquid phase selective hydrogenation of crotonaldehyde to crotyl alcohol production, the modified catalyst of Crotonyl alcohol showed a high reaction activity, high selectivity of crotonaldehyde conversion rate and stable. The In element is located in group III, the fifth cycle, the right side of the Sn element, and the Ge is in a diagonal position. We added Ge and Sn in TS-1 can improve the propylene epoxidation catalytic activity. So, we try to add In in the preparation process of the catalyst, as well as In in the TS-1 molecular for propylene epoxidation of supported Au catalysts.

\section{Experiment}

Preparation of Carriers. 30g prepared 0.05mol. $\mathrm{L}^{-1}$ hydrochloric acid in 500mL beaker; $37.4 \mathrm{~g}$ four ethyl orthosilicate (TEOS) with a dropping funnel drops of hydrochloric acid, room temperature drops below the magnetic stirring bordered 42g; isopropanol in $100 \mathrm{~mL}$ beaker; $0.58 \mathrm{~g}$ tetrabutylorthotitanate (TBOT) added, stirring for 5 minutes, then drop into the mixture on a step of adding finished stirring 15min; 8g four propyl ammonium hydroxide solution (TPAOH, 20\%), drops into the mixed liquor, adding finished form white colloid, white colloid aging 2h; will beaker 
white colloid smashing into the electric thermostat blast drying box $100^{\circ} \mathrm{C}$ dried overnight; the white solid powder transferred to the autoclave white lining, according to the mass ratio of TPAOH/ solid powder. Stir, then add TPAOH, crystallization in $1750 \mathrm{C} 24 \mathrm{~h}$; after crystallization of mixed liquid, centrifugal washing, drying, muffle furnace $500^{\circ} \mathrm{C}$ after removal of template: white powder obtained is required in our experiment TS-1 (100) molecular sieve.

Preparation of Catalysts. Firstly, we take the unfired TS-1 (100) molecular sieve, dipping it in a certain amount of $0.04 \mathrm{~mol} \mathrm{~L}^{-1} \mathrm{In}\left(\mathrm{NO}_{3}\right)_{3}$ solution, mixing $3 \mathrm{~h}, 8 \mathrm{~h}, 120^{\circ} \mathrm{C}$ dry impregnation, drying, roasting overnight $500^{\circ} \mathrm{C}$ prepared, In-TS-1 (100) carrier. Then the catalyst is denoted by alpha Au/ In-TS-1 (100). The beta alpha is the actual Au load mass fraction; the molar ratio of B for In and Ti, $\mathrm{Si} / \mathrm{Ti}$ is 100:1. The molar ratio of catalyst before reaction is not to preprocess.

\section{Results and Discussion}

XRD Characterization of Au/In-TS-1 Catalysts. Figure 1 shows the XRD patterns of $\mathrm{Au} / \mathrm{TS}-1(100)$ catalysts with different In doping contents. It can be seen in a part of Figure 1 that at the $7.8^{\circ}, 8.8^{\circ}, 23.2^{\circ}, 23.8^{\circ}, 45^{\circ}$, the strong characteristic diffraction peak occurs. In the b part of Figure 1, there are no split peaks at the $24.4^{\circ}$ and $29.4^{\circ}$. These indicated that the TS- 1 sample has a typical MFI structure and good crystallinity. There is no diffraction peak of Au in Figure 1, which shows gold particles were highly dispersed on the vector TS-1.
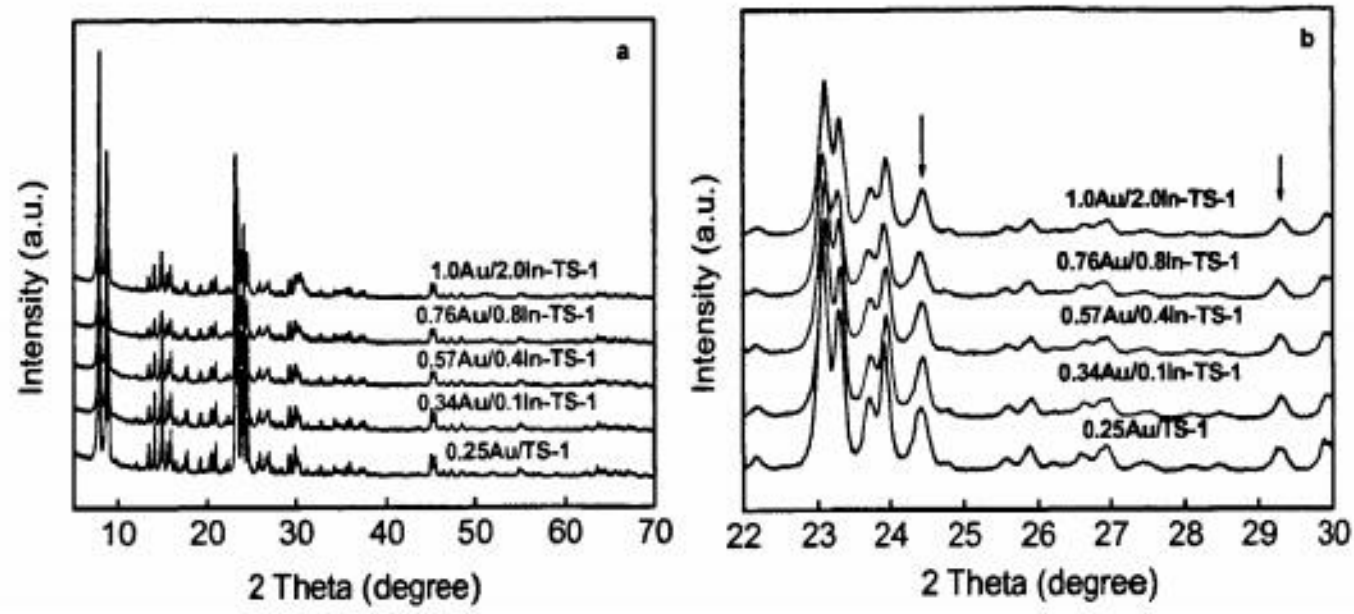

Figure 1 XRD patterns of Au/TS-1(100) catalysts with different In doping contents

UV-Vis Characterization of Au/In-TS-1 Catalysts. Figure 2 shows UV-Vis spectra of $\mathrm{Au} / \mathrm{TS}-1(100)$ catalysts with various In doping contents and pure $\operatorname{In}_{2} \mathrm{O}_{3}$. The power of $\operatorname{In}_{2} \mathrm{O}_{3}$ is roasted from $\mathrm{In}_{2} \mathrm{O}_{3} \cdot 5 \mathrm{H}_{2} \mathrm{O}$. We can see that the catalyst has strong absorption peak at 210nm, which is the characteristic peak of the tetrahedral coordination titanium species, and is also the important evidence for Ti to enter the molecular sieve framework. The absorption peak was not seen at 330nm, indicating that the carrier did not have the eight surface body coordination of the titanium species. no crystalline phase $\mathrm{TiO}_{2}$ exists. There is a very wide acromion map between $500 \sim 600 \mathrm{~nm}$. It can be attributed to the UV absorption peak of $\mathrm{Au}$. The $\mathrm{In}_{2} \mathrm{O}_{3}$ powder prepared by us has strong absorption at $345 \mathrm{nnl}$, and the In catalyst has weak absorption in the vicinity of $304 \mathrm{~nm}$. With the increase of In content, the peak is gradually strengthened. Therefore, we believe that the emergence of this peak is caused by the incorporation of In, compared with the powder of $\operatorname{In}_{2} \mathrm{O}_{3}$. There is a certain blue shift phenomenon. 


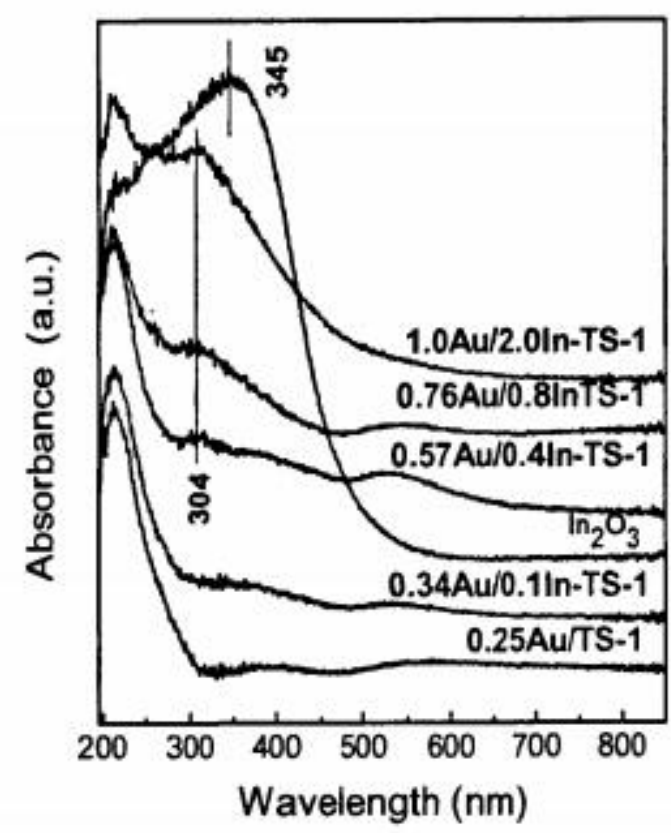

Figure 2 UV-Vis spectra of Au/TS-1(100) catalysts with various In doping contents and pure $\mathrm{In}_{2} \mathrm{O}_{3}$

TEM Characterization of Au/In-TS-1 Catalysts. Figure 3 shows the transmission electron microscopy characterization of Au/TS-1 (100) catalysts with different In doping content. From a part of Figure 3, we can see unmodified 0.25 Au/TS-1 (100) Au particles on the catalyst particle size is $2 \sim 8 \mathrm{~nm}$, particle size distribution and width; part b of Figure 3 shows the modified by In $0.57 \mathrm{Au} / 0.4 \mathrm{In}-\mathrm{TS}-1$ (100) Au particles dispersed on the catalyst is high, uniform particle size distribution that is about $\mathrm{L} \sim 2 \mathrm{~nm}$; c part of Figure 3 shows the higher content of In modified by 1 $\mathrm{Au} / 2.0 \mathrm{In}-\mathrm{TS}-1$ (100) catalyst, which was significantly higher than the number of $\mathrm{Au}$ particles in a and $b$ parts in Figure 3. The average particle size is $3 \mathrm{~nm}$, which can be observed in some agglomeration.
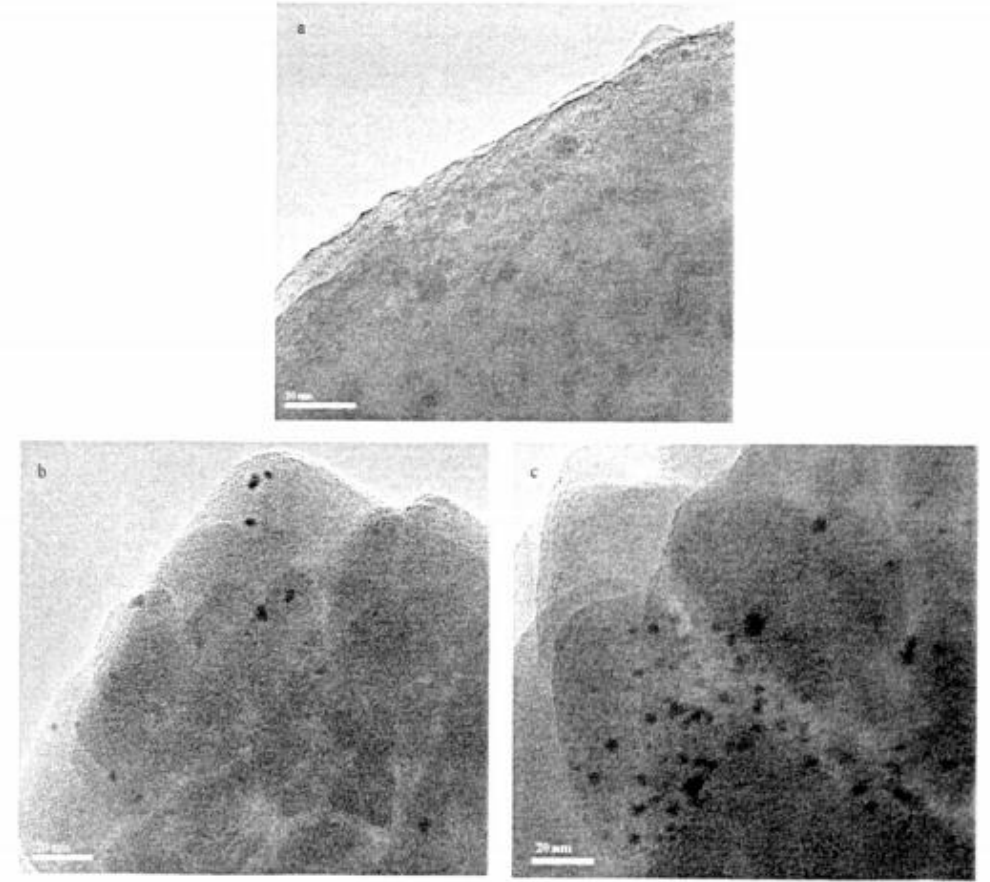

Figure 3 TEM of images of (a) 0.25 Au/TS-1(100), (b) 0.57 Au/0.4In-TS-1(100), (c) 1.0

$\mathrm{Au} / 2.0 \mathrm{In}-\mathrm{TS}-1(100)$ 
Stability Testing of Au/In-TS-1 Catalysts Activity. Figure 4 shows the stability results of $0.25 \mathrm{Au} / \mathrm{TS}-1$ (100) and $0.57 \mathrm{Au} / 0.4 \mathrm{In}-\mathrm{TS}-1$ (100) of propylene epoxidation catalyst. The reaction can be obtained from Figure 4, the $0.25 \mathrm{Au} / \mathrm{TS}-1$ (100) catalyst. The initial propylene conversion rate was $3.4 \%$, stable down to $2.0 \%$, the catalyst activity decreased rapidly. $0.57 \mathrm{Au} / 0.4 \mathrm{In}-\mathrm{TS}-1$ (100) catalyst in propylene conversion of initial reaction rate was $4.2 \%$, stable to $3.8 \%$, significantly higher than the former has better stability. In addition, 0.25Au/TS-1 (100) catalyst selectivity of PO is at around 87\%, which is higher than $0.57 \mathrm{Au} / 0.4 \mathrm{In}-\mathrm{TS}-1$ (100) catalysts (81\%). However, the gold catalyst in propene epoxidation in general, exist the phenomenon of inactivation. So the stability margin of catalyst is the key problem of industrial production. The research work shows that the use of In modified TS-1 as the carrier can effectively enhance the stability of the Au catalysts.
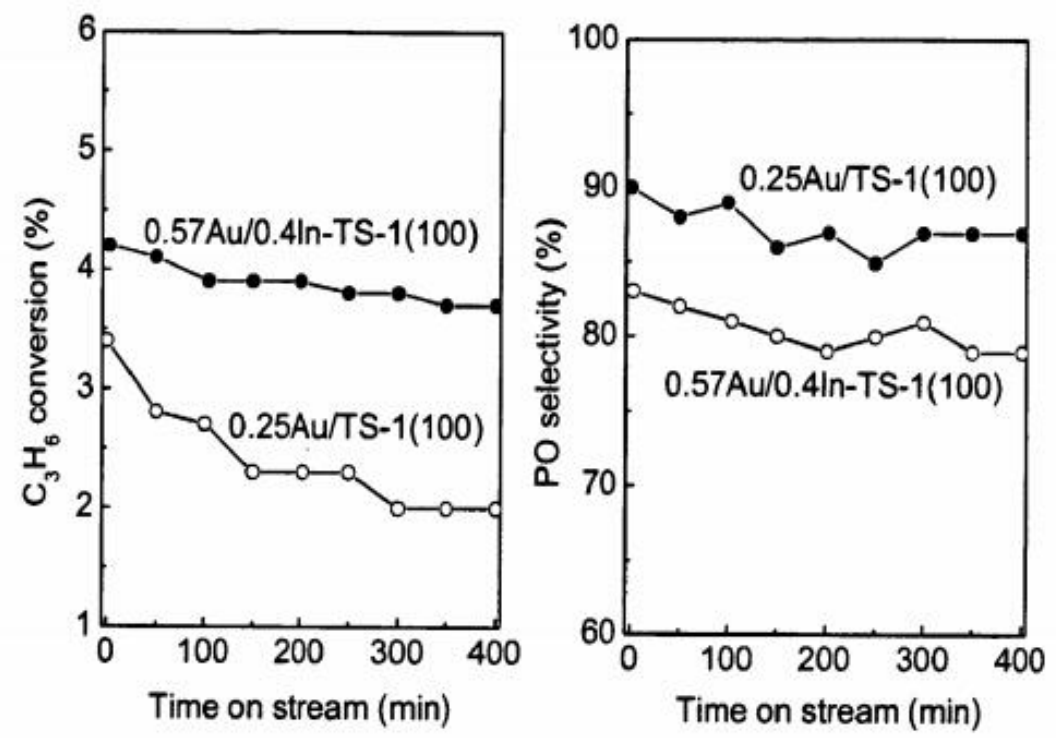

Figure 4 Epoxidation of propylene on $0.25 \mathrm{Au} / \mathrm{TS}-1(100)$ and $0.57 \mathrm{Au} / 0.4 \mathrm{In}-\mathrm{TS}-1(100)$ catalysts

\section{Conclusion}

The characterizations of XRD and UV-Vis indicate that the TS-1 synthesized in this study has the MFI structure, and the Ti species of tetrahedral coordination has entered into the framework of the molecular sieve. The addition of In increases the load and the dispersion of Au, and reduces the $\mathrm{Au}$ particle size, which inhibits the aggregation of Au. It may be the main reason for the improvement of the catalytic activity.

\section{Acknowledgements}

This research was financially supported by Youth Fund of National Natural Science Foundation (Grant No. 21506170).

\section{References}

[1] Zhang Wenmin, Liu Yiwu, Li Hao, Li Xiaoqiang, Zhang Xiaoming, Journal of Molecular Catalysis (China), Vol. 25(2011) No 3, p.213-219

[2] Li Yichuan, Shen Benxian, Zhao Jigang, Journal of China University of Petroleum (Edition of 
Natural Science), Vol. 36 (2012) No 6, p.177-182

[3] Zhang Chao, Pan Xiaorong, Luo Mengfei, Lu Jiqing, Journal of Zhejiang Normal University (Natural Sciences), Vol. 35 (2012) No 3, p.305-310

[4] Wang Huan, Tianjin Chemical Industry, Vol. 30 (2016) No 5, p.34-37 